\title{
Improving fluid balance monitoring on the wards
}

Sobanakumari Jeyapala, Alice Gerth, Aarti Patel, Nazia Syed

United Kingdom

\begin{abstract}
Clinical experience and nursing metrics have consistently identified poor documentation of fluid balance monitoring at Milton Keynes University Hospital, compromising patient safety and quality of care. This project aimed to increase the percentage of fluid balance charts correctly completed on the wards.
\end{abstract}

Three areas for improvement were identified: understanding the importance of good fluid balance monitoring, correct identification of patients requiring monitoring, and ease of completion of fluid balance charts. Three interventions were deployed on two acute medical awards in consecutive cycles; 1) small group education for staff, 2) creation of board magnets to aid the multidisciplinary team to identify patients requiring monitoring, 3) modification of the current fluid balance chart. Questionnaires were utilised to highlight improvements with current charts and measured staff awareness pre and post education. Each intervention was implemented for one week followed by daily surveys for four days to monitor compliance.

Initial results showed a range of $6-12$ charts used daily per ward. Of these $0-45 \%$ of them were correctly filled. Post education there was a reduced number of inappropriate charts. Introduction of board magnets improved correlation between doctors and nurses in identification of patients (52\% before, $77 \%$ after magnets). Following modification there was a subjective improvement in the quality of chart completion.

This study highlighted that understanding and use of fluid balance monitoring can be improved for nurses, health care assistants (HCAs), and doctors. These improvements allow better documentation and safer patient care. As a result, Milton Keynes University Hospital is investing in magnets and modified charts for a Trust-wide pilot.

\section{Problem}

Fluid balance assessment on busy ward rounds or during general patient review requires clear documentation of fluid input and output. This allows accurate assessment of a patients' fluid balance status, which is pertinent when reviewing intravenous fluid prescriptions or assessing for early clinical deterioration [1].

Anecdotal evidence from junior doctors in a district general hospital (DGH) in England highlighted improper fluid balance monitoring largely due to poor documentation on fluid balance charts. Patients not requiring monitoring had charts at their bedside, increasing the work load for nurses. This consequently impacted on the overall quality of fluid balance charts for patients in need of accurate fluid balance monitoring.

The charts were A3 sized and cumbersome, with little guidance on correct chart completion (figure 1). They were commonly underfilled with either fluid input or output missing. Patient details and indication for the chart were often not documented. Uncommon abbreviations e.g. "PU'd" for passed urine were used as a substitute for numerical values.

\section{Background}

The hospital nursing metrics highlighted that fluid balance chart documentation was sub-optimal on all wards outside of ICU [2].
This was also noted in the 2015 CQC report stating that the quality of fluid balance recording varied throughout the hospital [3]. Reid (2004) reported the main reasons for fluid balance charts being poorly completed as staff shortages, lack of time and inadequate training [4]. Therefore, any interventions to improve fluid balance documentation need to account for these factors.

Our initial survey to assess the ease of completing fluid balance charts was scored out of 10 , with an average score of 7.4 but ranged from $3-10$. The upper end of this range is likely due to familiarity with the charts by established nursing staff; a chart that is easy to complete on first encounter (e.g. by student nurses and agency staff) would be preferable. Initial data collection also highlighted poor correlation (52\%) between doctors and nurses when identifying patients requiring fluid balance monitoring. This discord reduces the ability of the multi-disciplinary team (MDT) to provide good clinical care.

\section{Baseline measurement}

Data was collected to assess 5 baseline measurements:

1. A questionnaire assessed nursing and health care assistant understanding of fluid balance monitoring - mean quiz score (out of 5) prior to education 1.45 (S.D. 1.17)

2. Correlation between nurses and doctors identifying patients requiring monitoring - mean baseline correlation $52.5 \%$ (S.D. 28.52) 
3. Identifying the number of charts on the wards (approximately 27 beds) to assess nursing work load - mean baseline measurement 9.3 charts (S.D. 4.47)

4. A questionnaire assessed the ease of completion of current charts (see figure 1) (Score of $10=$ easy to complete) mean baseline measurement 7.4 (S.D. 2.42)

5. Assessing the percentage of charts correctly filled out (criteria: patient name, indication for fluid balance monitoring, a recording at least once every 4 hours, totals) mean baseline measurement $25.4 \%$ (S.D. 1.68)

See supplementary file: ds6236.pdf - "Figure 1: Current Fluid Balance chart"

\section{Design}

Three key areas for improvement were identified following discussions with senior doctors, junior doctors, and nursing staff:

1. Understanding the importance of fluid balance monitoring

2. Improving recognition of the patients which require monitoring amongst the MDT

3. Correct completion of fluid balance charts

When reviewing these areas several interventions were devised and the feasibility of implementing each option was discussed with the relevant ward managers and nursing staff. This provided valuable insight into problems and allowed selection of 3 PDSA (Plan, Do, Study, Act) cycles which could be implemented on the wards (see figure 2 - highlighted boxes).

Group sessions providing education on fluid balance monitoring was key to addressing all 3 areas. However challenges such as high staff turnover and shift patterns highlighted the need to provide written information, which we felt would be most accessible placed on the back of the fluid balance charts.

Recognition of patients requiring monitoring was best addressed through the use of a simple visual aid. The use of labels on patient jugs could provide reminders to document fluid balance during routine observations. The use of magnets to be placed on handover boards is a low-cost intervention and allows all members of the team to recognise patients quickly, while additionally providing a gentle reminder to discuss fluid balance monitoring during handover.

Questionnaires were used to highlight preferences and dislikes regarding the current chart and the results prompted the creation of a modified chart, with guidance on accurate documentation (see figure 3). The key requirements of the chart were discussed with the renal and intensive care physicians. This pilot chart was created with the intention of identifying whether changing the current charts could allow better practice, however long term change would require formal discussion through clinical governance and practice development with an extended pilot period.

Following discussion with the heads of nursing these 3 PDSA cycles were implemented on 2 acute medical wards with well- established nursing bases with the crucial assistance of the ward managers. Each cycle lasted 2 weeks with 1 week for implementation and 1 week for data collection.

\section{Strategy}

PDSA cycle 1: Informal group education sessions with nursing staff and HCAs were carried out over 4 consecutive days during afternoon handover. The sessions lasted 10 minutes and covered the reasons for fluid balance monitoring and what was considered appropriate completion of a fluid balance chart (e.g. calculating cumulative balances at minimum every 4 hours, avoiding abbreviations such as PU'd for passed urine etc.). Supplementary written information was provided (see figure 3). A before and after quiz was used to assess the effectiveness of the sessions. It was found that multiple sessions were required each day as not all staff could be off-the ward at the same time. The following week data was collected on both wards to assess for any change.

PDSA cycle 2: Paper labels for patient jugs were created, however it was noted that labels required changing every time a water jug was changed, which was at minimum daily and some 'nil by mouth' patients could not have jugs. There were also concerns surrounding the hygiene of paper labels. As a result this intervention was not piloted. The use of laminated blue tacked labels to be placed on the handover board were simple to implement. This consequently allowed fluid balance to be discussed during "board rounds" and meant only patients with board labels had fluid balance charts filed, thus reducing unnecessary work for nursing staff.

PDSA cycle 3: Using the valuable feedback from nursing staff and HCAs a modified A4 fluid balance chart was created (see figure 3). This chart has clearly denoted boxes for 4-hourly cumulative totals, which would coincide with routine observation times. The written information supplementing the teaching sessions can be located on the back of the charts so that it could be accessed at all times. The normal volume of fluid in jugs, cups, and mugs was also visually depicted for ease of estimation of fluid intake. This information was relevant particularly for student and locum nursing/HCA staff that were not present during teaching sessions.

\section{Results}

In the week following each PDSA cycle, data was collected over a 4-day period, to assess the effectiveness of each intervention. The same 5 baseline measurements were reassessed. Baseline 1, 2, and 4 were only assessed following PDSA Cycles 1,2 , and 3 respectively, the remaining baseline measurements were reviewed following every cycle:

1. Following PDSA cycle 1: Nursing and HCA understanding of fluid balance - mean pre-education quiz score (out of 5 ) = 1.45 (S.D. 1.17). Mean post-education quiz score $=2.6$ (S.D. 1.17). The desired improvement was not found following education sessions (see figure 4).

2. Following PDSA cycle 2: Correlation between nurses and doctors identifying patients requiring monitoring - mean 
initial correlation $52.5 \%$ (S.D. 28.52). With the introduction of board magnets this improved to $77 \%$ (S.D. 22.8) (see figure 5).

3. The total numbers of charts on the wards (approximately 27 beds) were assessed after each cycle - mean initial measurement 9.3 charts (S.D. 4.47). This was significantly reduced following the introduction of board magnets to 7.0 charts (S.D. 2.16) (see figure 6). When collecting data it was noted that the number of unnecessary charts was reduced, thus reducing nursing workload.

4. Following PDSA cycle 3: Ease of completion of charts (a score of $10=$ easy to complete) - mean initial measurement with the current chart 7.4 (S.D. 2.42), with the introduction of a modified pilot chart this increased to 8.1 (S.D 0.83). The mean was not significantly increased, however the S.D. was much smaller. This suggests that the chart was easy to complete regardless of its familiarity (see figure 7 ).

5. Assessing the percentage of charts correctly filled out showed an initial mean measurement of $25.4 \%$ (S.D. 1.68). The end mean was $28.4 \%$ (S.D. 1.35) (see figure 8). This was also not a significant improvement. However subjective analysis showed that although the charts did not meet all of the criteria required, the quality of completion had improved e.g. only 1 of the 4 criteria were absent, rather than 2 or 3 .

See supplementary file: ds6364.pptx - "Figure 2, Figure 3, Figure 4, Figure 5, Figure 6, Figure 7, Figure 8 "

\section{Lessons and limitations}

Following this project 3 main areas for learning can be identified. Firstly, we would have greatly benefited from having junior nurses as part of the team. This would increase communication between doctors and nursing staff, allow greater nurse involvement when designing interventions and improve implementation of interventions on the wards.

Secondly, it is key to consider interventions that are practical, lowcost, and that comply with hospital policy. The use of stickers on jugs contravened hospital hygiene policies and was too costly to implement as new stickers would permanently need to be generated whenever jugs were changed. In comparison, the board magnets were very cost effective as they could be re-used for new patients.

Finally, this project highlighted that as junior doctors we are capable of initiating positive change. This project was presented to the chief executive and senior nursing staff. They were enthusiastic about carrying the project forward. Following this meeting the trust is currently investing in magnets for handover boards and considering using the concept of our fluid balance chart (including the educational information on the reverse) as a pilot for modifying the current chart.

\section{Conclusion}

This project identified the weaknesses of the current fluid balance chart and general fluid balance monitoring. It showed that understanding and use of fluid balance monitoring could be improved for nurses, HCAs, and doctors. The use of board magnets is a relatively low financial investment that can allow the MDT to provide effective clinical care. The trust is now planning to review its fluid balance monitoring practices and use our ideas and modified fluid balance chart as the basis for future change.

\section{References}

1. NICE Guidelines (CG174) - Intravenous fluid therapy in adults in hospital (last accessed 17/09/15)

2. Milton Keynes Hospital Nursing Metrics 2014.

3. Care and Quality Comission. Milton Keynes Hospital NHS Foundation Trust, Quality Report available at www.cqc.org.uk/sites/default/files/new_reports/AAAA3021.p df (last accessed 17/09/15)

4. Reid J, Robb E. Improving the monitoring and assessment of fluid balance. Nursing Times. 2004; (100: 20): 36-39.

\section{Declaration of interests}

None

\section{Acknowledgements}

Dr Joy Halliday - QIP mentor.

Dr Ram - Renal Physician.

Emma Codrington - Head of Nursing Medicine.

Glen Warren - Hospital ward manager.

Laura McGeary - Hospital ward manager.

\section{Ethical approval}

This was an improvement study, not carried out directly on human subjects and as such no ethical approval was required. 